\title{
Polyphase Drive Systems Configuration aiming EMI Mitigation
}

\author{
G. Winnischofer ${ }^{1}$ and C.A.F. Sartori ${ }^{1,2}$ \\ ${ }^{1}$ Laboratory of Applied Electromagnetism LMAG - PEA/EPUSP \\ Electric Energy and Automation Engineering Department \\ Polytechnic School of the University of São Paulo- Brazil \\ Phone/Fax number:+0055 11 30915124, e-mail: godofredo.winnischofer@usp.br, sartori@pea.usp.br \\ ${ }^{2}$ Nuclear Energy Research Institute / National Nuclear Energy Commission - IPEN/CNEN-SP \\ e-mail: $\underline{\text { sartori@ipen.br }}$
}

\begin{abstract}
Topologies of motor drive systems are studied, aiming the reduction of common-mode (CM) currents. Initially, the aspects concerning the $\mathrm{CM}$ currents circulation are analysed. The reason of common-mode voltages generation, the circulating paths for the resulting CM currents and their effects are discussed. Then, a non-conventional drive system configuration is proposed in order to reduce the CM currents and their effects. This configuration comprehends a non-conventional inverter module wired to a motor with an unusual connection. The cables arrangement differs from the standard solution, too. The proposed topology is compared with other ones, like the active circuit for common-mode voltages compensation. The contribution of the configuration to the reduction of $\mathrm{CM}$ voltages and currents and their related interferences are evaluated, based on numerical simulations. Some results are presented and discussed regarding the suitability of the proposed configuration as a potential solution to reduce the CM currents effects, when the state of art and implementation cost of drives are taken into account.
\end{abstract}

\section{Key words}

Electromagnetic compatibility (EMC), electromagnetic interference (EMI), common-mode, motor drive system.

\section{Introduction}

\section{A. General aspects of drive systems technologies}

The cost reduction of inverters allowed them to be widely used to control induction motors in many applications, mainly in industry and transportation segment. The energy saving and mechanical stress reduction are usually mentioned among the benefits of variable speed drives applications. The use of IGBTs is one of the main responsible for the inverter popularization. The high-speed commutation of IGBTs and the consequent reduction of switching losses and inverter module size are among the main benefits of the application of this power device technology. Moreover, it also allows that the inverters operate at higher switching frequencies, which improves the drive system performance at the same time that contributes to the reduction of motor losses. On the other hand, they are also responsible for electromagnetic disturbances [1]. Thus, due to the growth of number of inverters in a variety of environments, the importance of the control of electromagnetic interference (EMI), in particular the ones originated due to CM currents, tends to be critical.

The high-speed commutation of IGBT's, that contributes to a better response of the driven motor, also produces transients of voltage and current, which are responsible for several electromagnetic issues, such as reflected wave overvoltage. The control techniques based on PWM, which is probably the most common, and the corresponding drive system topology contribute to stress the effects of electromagnetics phenomena concerning the common-mode and differential-mode voltages. That is, they are responsible for conducted and radiated electromagnetic interference (EMI). Apart from the electromagnetic interference, these events play an important role in reliability of the drive system. Many failures may be attributed to these effects. Among them, motor insulation stress due to overvoltages and the bearings wear due to common-mode current flow can be mentioned [1]-[8].

Over the last 20 years, an extensive literature was generated, discussing these problems. Also, it can be stated that many advances have been made to address them. Reinforced motor insulation and cable length adequacies were established as requirements to avoid overvoltage stresses. Also, isolated bearings became a common and special practice for inverter driven motors. The using of shielded cables, with an ideal phase distribution, specially developed for inverter driven systems, can also be mentioned as an engineering practice. Some of these proposed countermeasures help to minimize EMI, but, along these years, the main focus was on the functional system reliability aspects. Based on this 
scenario, we can state that exists a large field for EMI improvement in inverter driven systems. Thus, this study focuses on this point, in particular, on the control of emissions caused by common-mode noises.

\section{B. Drive systems configurations and EMI control}

The most common topology for drives is the three-phase inverter bridge with six IGBTs powered by a six-pulse rectifier. Since the VSI (Voltage Source Inverter) configuration is the preferred one, rectifier and inverter are connected through a capacitor bank that forms the DC link. The rectifier is connected to a common three-phase supply system; and the inverter bridge is connected to the three-phase motor. On the supply side, the most of the electromagnetic interference, including the harmonic effects, may be attributed to the distortion of the current waveform due to the rectification. For this reason, twelve and twenty four pulses rectifiers have been considered as alternative for harmonic content reduction. Note that the measures taken to control conducted emission are also effective ways to control the radiated emissions and its resulting EMI.

At the other end, in the inverter side, the object that is directly affected by conducted emission is the driven electric motor. Although it has a high EMI immunity, the emissions can be transmitted to other systems through a capacitive and inductive coupling or to be radiated, affecting other devices. As aforementioned, the pulses generated by IGBT commutation are responsible for high frequency harmonics content. Thus, the high frequency voltages produce high frequency currents that flow through the existing stray capacitances dispersed in motor windings and cables. Those currents are the differentialmode (DM) and the common-mode (CM) currents [1]-[3], [6]-[8].

The differential-mode currents are the ones that flow back to the noise source through the same circuit as the fundamental current. Due to this reason, certain authors call them as normal-mode currents, too [2]. The resulting electrical fields created by those currents are subtracted by each other, resulting in a reduced electric field. The common-mode currents are the ones that flow back to the noise source through the ground. As a consequence, the resulting electric fields are less compensated and the $\mathrm{CM}$ currents produce more intensive electrical fields. Therefore, since common-mode currents have much more potential for producing EMI due to radiated emissions than differential-mode currents, the former is usually the focus of the investigation [2],[9].

In three-phase drive systems, common-mode voltages are caused by the asymmetry of the pulses. As shown in Fig. 1 , the common-mode voltage can be obtained simply as the average of the three-phase voltages. In a perfect sinusoidal three-phase system, the result will be zero, which means, no common-mode voltage. However, when motor is fed by a PWM inverter, the supply voltage is not sinusoidal, but composed by discrete pulses. And then, the result will be different from zero [5]-[8],[10].

$$
V_{c m}=\frac{V_{a}+V_{b}+V_{c}}{3}
$$

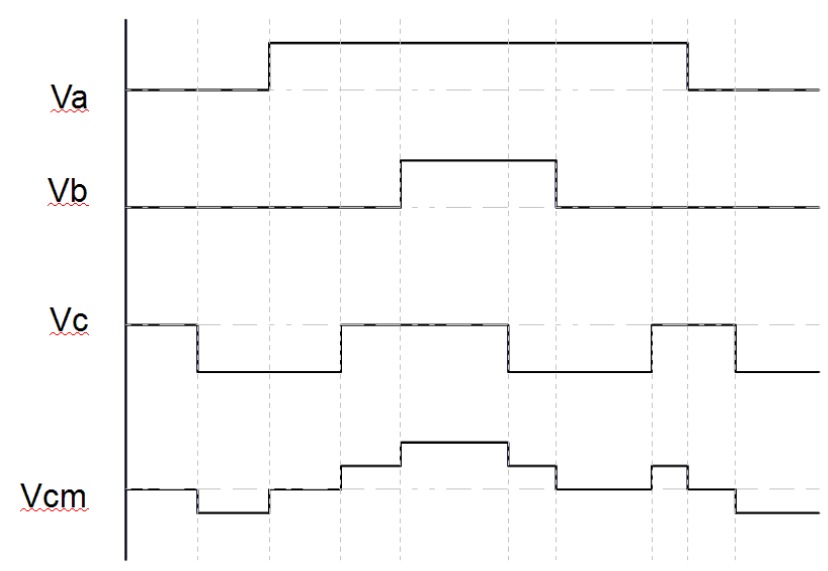

Fig. 1 - Pulse voltage waveforms at motor three phases, fed by an inverter, and the resulting CM voltage.

The most common technique to control common-mode voltages is the use of passive filters at the inverter output. The filters can be a combination of chokes with resistors, capacitors and inductors, the well-known RLC filters. The RLC filter has the advantage to reduce the differentialmode and common-mode voltages at the same time. They also contribute to reduce the reflected overvoltage, when long cables between inverter and motor are used. However, they are not considered efficient for all the frequency ranges. Furthermore, the addition of filters results in increased weight and space consumption. Also, losses to the system are added and costs are increased [1],[3],[5].

The active circuits for common-mode voltage cancellation are also widely proposed in the literature, as a potential solution for common-mode voltages reduction. However, they are not commonly found in the market. They imply in the increase of the number of power semiconductors. A common topology is the four-leg configuration. In this topology, a fourth pair of IGBT's is added to the inverter module and a common-mode transformer is wired between the inverter and the motor. This circuit captures the $\mathrm{CM}$ current at the output of the inverter and lead it back to the internal DC link. Those circuits have the advantage of reducing common-mode currents in a broader range than passive filters. Comparing to the RLC filters, they will have a lower size and weight. However, they also demand extra devices. As disadvantage of those active circuits, it can be mentioned the control complexity and the reduction of reliability, due to the increase of failure probability rate. Many authors present a variation of the configuration, based in the same principle [5],[8].

\section{The Proposed Configuration}

In this paper, it is proposed a new solution, based on the active circuits principles, of capturing and deviating the CM current back to the source, but presents some particularities like a simpler control and the role that the 
topology and physical arrangement take in process. The idea is simple: assuming that the asymmetry of the pulses causes the common-mode voltages, then a symmetrical configuration shall mitigate them. For each phase $\mathrm{Va}, \mathrm{Vb}$ and $\mathrm{Vc}$, phase voltages $\mathrm{Va}^{\prime}, \mathrm{Vb}^{\prime}$ and $\mathrm{Vc}^{\prime}$ are added, in order to cancel the common-mode voltage. The Fig. 2 illustrates this approach.

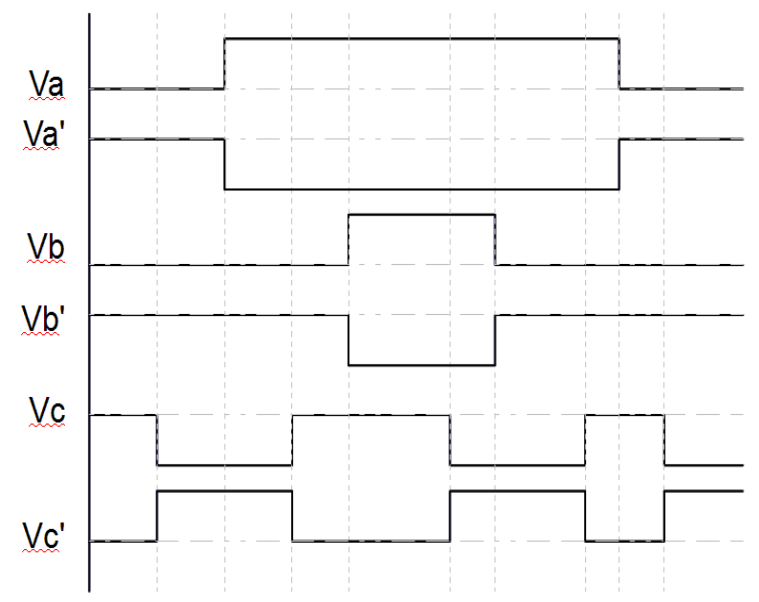

Fig. 2 - Voltages at "double three- phase topology" used to CM emission control.

Because six voltage signals are necessary, we get a sixphase system. However, since three of the phases shall necessarily be the mirrored image of the other three ones, a term which also may be used is double-three-phase system. At least in some cases, as it will be discussed later, this term will better define the configuration.

The Fig. 3 presents the proposed configuration. The inverter is composed by two conventional three-phase
IGBT bridges. There are two possibilities to use such inverter to drive an induction motor: with a six-phase motor or with a three-phase motor with a pair of coils for each phase, turning it a double-three-phase motor. What differs from one alternative to the other is the control of IGBT's firing. In a six-phase inverter, the switching of each phase is shifted by sixty degrees from the previous one. In the double-three-phase inverter, there are two sets of three-phase inverter. In each inverter, each phase is shifted by 120 degrees from each other, but the second three-phase inverter is shifted by 180 degrees from the first one, as indicated in fig. 3 .

The possibilities for the induction motor are as following: a) a six-phase motor with the windings connected in ring, as shown in Fig. 4a; b) a six-phase motor with the windings connected in star, as shown in Fig 4b; c) a double three-phase motor in a double-delta connection, as shown in Fig 4c; d) a double three-phase motor in a double-wye connection. For the latter, since the center of both connections is common, the end configuration will be like the one shown in Fig $4 \mathrm{~b}$.

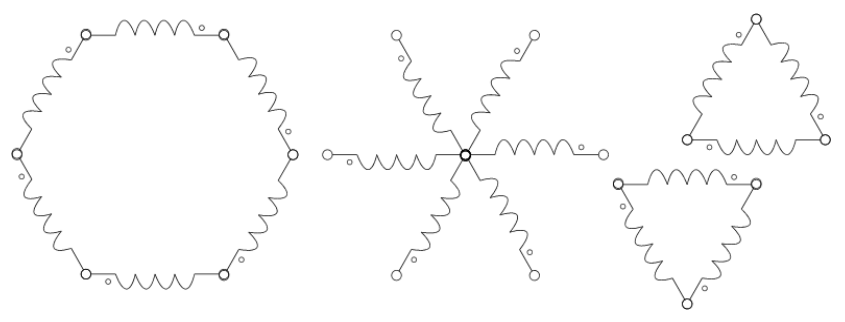

Fig. 4 - Motor connections: a) six-phase ring (left), b) six-phase star or double-three-phase wye (center) and c) double-threephase delta (right).

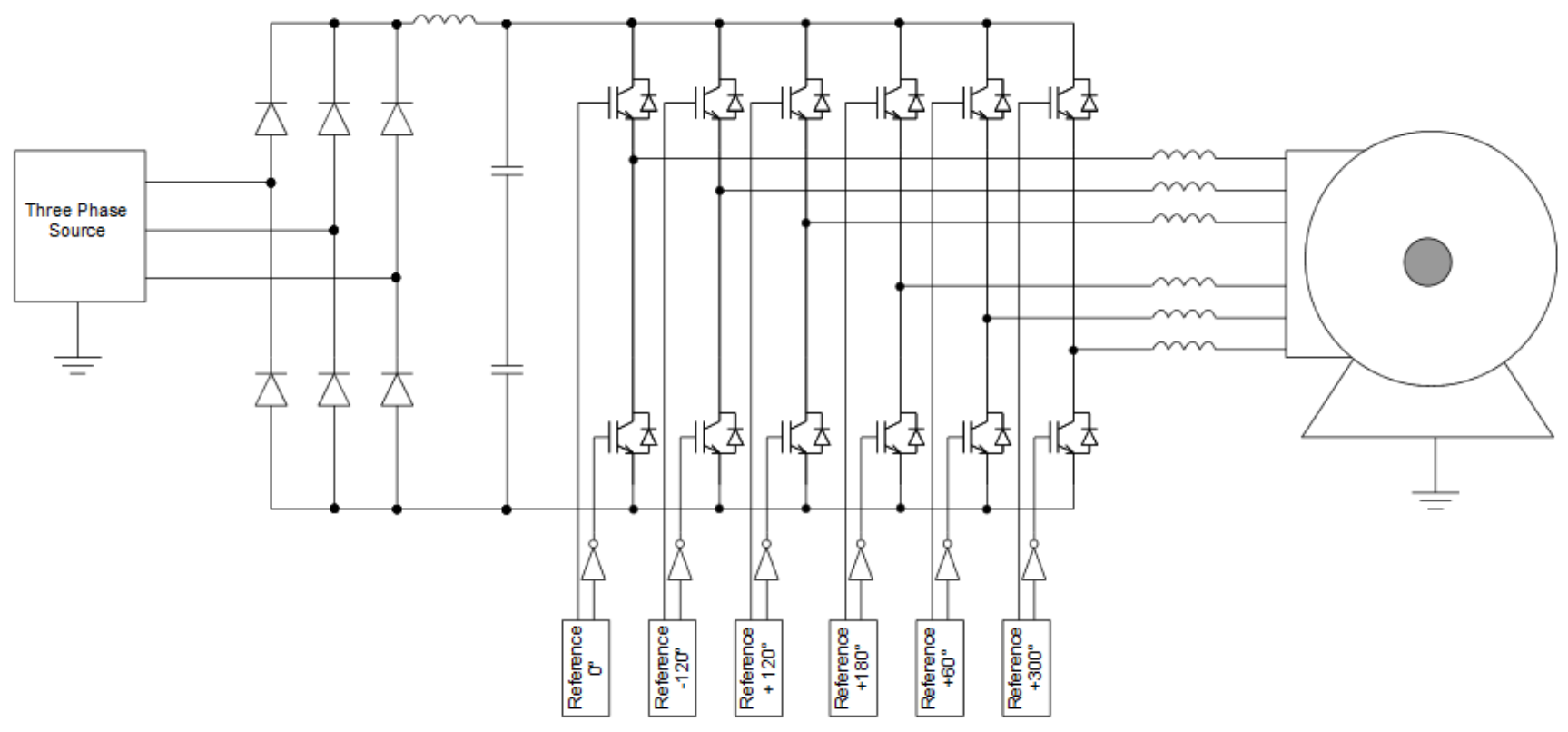

Fig. 3 - Proposed Double Three-phase (Six-Phase) Inverter Topology. 
The three-phase motor with double winding is available in the market as a conventional twelve terminals induction motor. The motors of this type are manufactured for several voltage connections such as 220/380/440V, as shown in Fig. 5. It is feasible by making a new winding connection, inverting the polarity of the second set of three-phases, to reach the desired configuration.
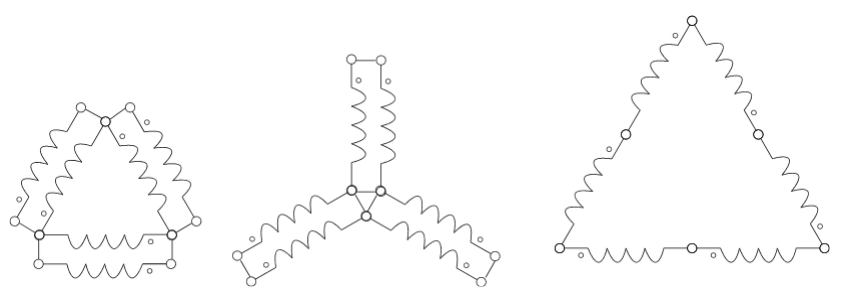

Fig. 5 - Possible connections of a twelve terminals motor: $220 \mathrm{~V} / 380 \mathrm{~V} / 440 \mathrm{~V}$.

One simulation model was generated for each motor configuration described above. As an example, the circuit model shown in Fig. 6 was implemented as double-delta motor model.

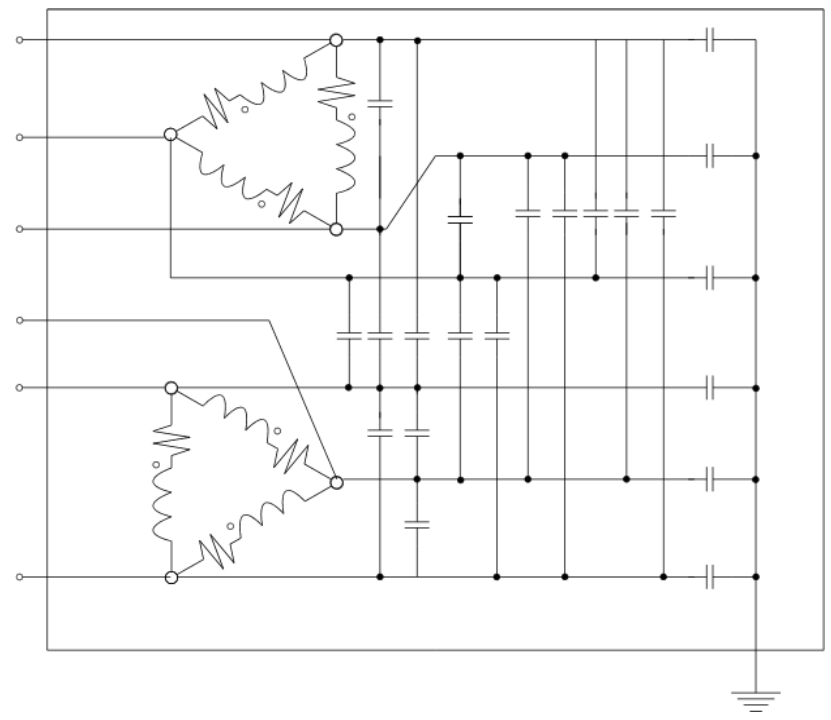

Fig. 6 - Double-delta motor circuit model.

Also, one simulation model was generated for each control alternative of the inverter shown in Fig. 3. Then, the several combinations of inverter control and motor models were tested in a circuit simulator. Some results are presented in the next item. In this step of the investigation, the relations between the results obtained in each simulation are evaluated as sensitive cases, in order to check its potentiality. So, preceding to these models, two conventional three-phase drive system, with a delta and wye connected motor were generated for comparison reference purpose.

\section{Results}

The models of conventional three-phase drive system were simulated as starting point reference. The Fig. 7 shows the waveforms of the currents that circulate through the three phases and through the ground (common-mode). This currents trend concerns the motor in delta connection. Similar curves were obtained for a threephase wye connected motor.

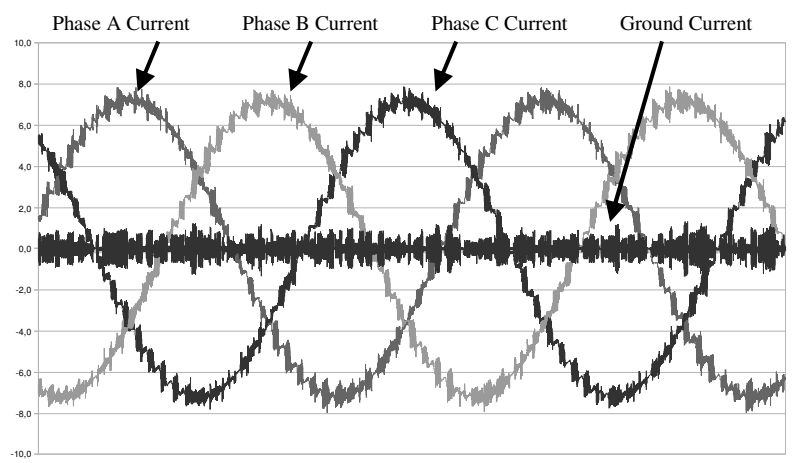

Fig 7 - Phases and ground currents in a conventional three-phase delta connection motor topology.

It can be seen that the three phases current waveforms have a high frequency harmonic over their fundamental and there is visible that a current is circulating through the ground, the common-mode current. In case of the wye connected motor, higher amplitude was reached by the ground current, due to the lesser impedance.

Later, the proposed solution was simulated. As mentioned before, it was done in several combinations: a doublethree-phase motor, in wye and later in delta connection, driven by the double-three-phase inverter and a six-phase motor in a ring connection and later a six-phase motor in star connection driven by a six-phase inverter. The results obtained with the double-three-phase inverter driving a double-delta motor were selected to be shown. The Fig. 8 shows the current waveforms from three of the six phases and from the common-mode (ground) current. Although the results obtained for the other proposed configurations are not here presented, it must be mentioned that they are similar. Also, the other three phases current waveforms not displayed in the graph are similar to the displayed one, just mirrored (displaced by 180 degrees). In this picture, it can be seen that the common-mode current is near zero value.

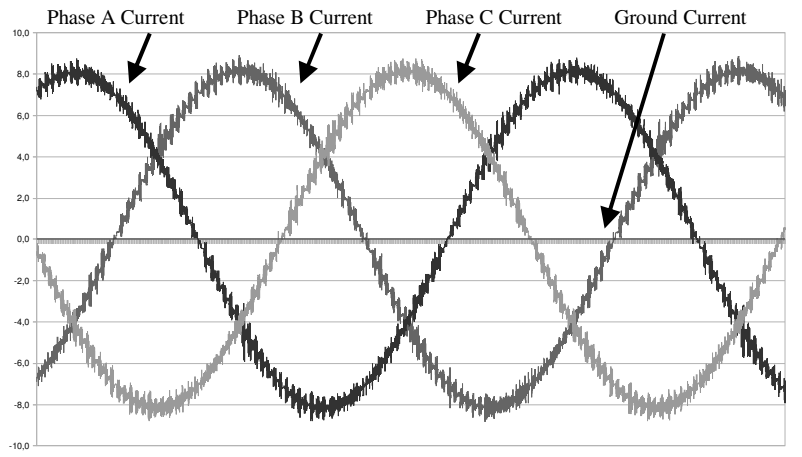

Fig 8 - Phases and ground currents in the proposed topology, corresponding to Fig. 3 for inverter and Fig. 6 for motor. 
It is notorious that the current that is flowing through the ground, that means, the common-mode current, has much higher amplitude in the conventional configuration than in the proposed topology. This current, when flowing through the motor bearings, is responsible for its erosion. Also, this current flowing through the grounding cabling is responsible for interference in other equipment. It is noticed also that the harmonic components over the fundamental waveform are inferior in the proposed solution.

The previous results lead us to believe that the proposed topology can be considered as a potential solution to control the CM emission. The common-mode current was substantially reduced without the addition of heavy filters. Regarding to the physical issues, it must be noted that the proposed solution imply in the increase of the number of power semiconductors, as well in the number of cable leads between drive and motor. This will be discussed in the next section with an economical analysis.

Furthermore, in order to take into account the influence of the installation, the circuit model was improved. Firstly, it was considered an installation between drive and motor composed by two three-phase cables, developed for applications with conventional inverters. The result of the simulation is shown in Fig. 9.

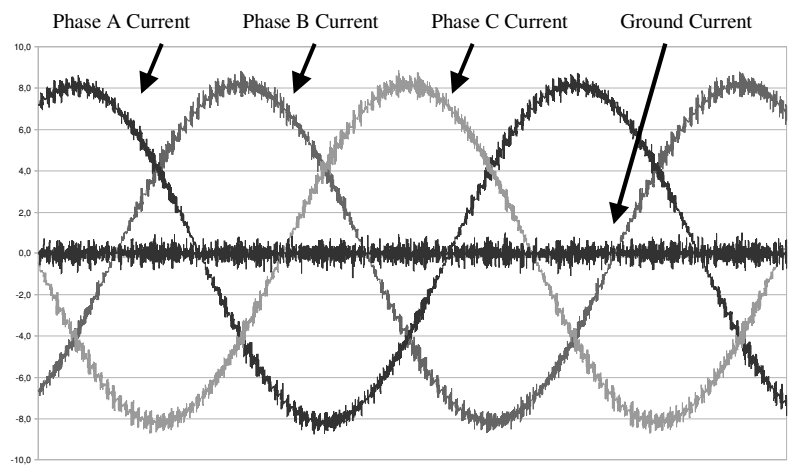

Fig 9 - Phases and ground currents in the proposed topology with the connection between inverter and motor through 2 conventional three-phase cables.

Note, that in this new simulation, the common-mode current is present. It flows through the cables stray capacitances. The reason for this behaviour is that the asymmetry of the three-phase system remains in the cables.

Another model, considering the motor powered through three pairs of cable was then implemented. The result of this simulation is shown in Fig. 10. The arrangement for this configuration is the following: each pair of motor phases composed by the phases shifted by 180 degrees is fed through one pair of cables. In this new simulation, the symmetry obtained with the proposed configuration is kept in the installation between the inverter and motor and the reduction of common-mode current is obtained again.

These two later simulations shown the importance that the installation have in this process. This concern is not new.
Installation is one of the major responsible for electromagnetic interference in a drive system. However, the intention is to highlight another role that the configuration of the installation can take: usually, the main focus is given to the characteristics of cable and physical arrangement to reduce radiated EMI and electromagnetic coupling with other installations; instead of this, here the intention is to focus on the contribution of the cable configuration to the natural mitigation of noises, reducing the potentiality of the system in generating EMI. Also, it shall be observed that the best installation practices for a conventional architecture may be inadequate practices for the proposed solution and viceversa.

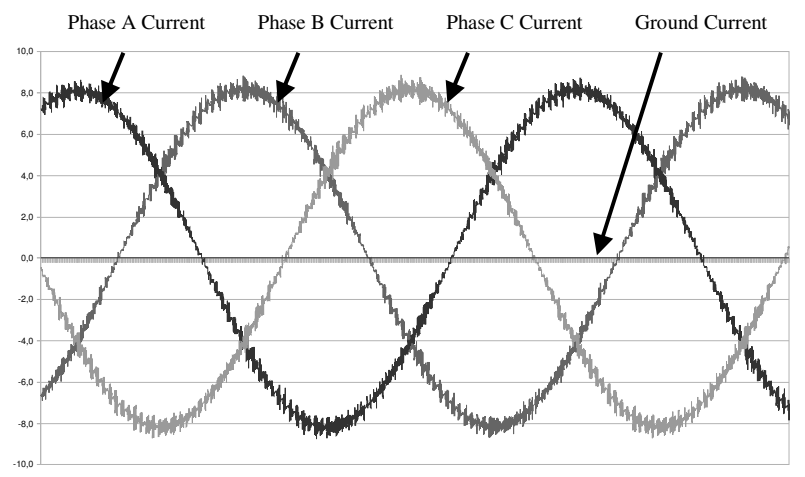

Fig 10 - Phases and ground currents in the proposed topology with the connection between inverter and motor through 3 pairs of cables.

\section{Conclusion}

The simulations have shown that the proposed doublethree-phase configuration can be suitable for EMI reduction acting upon common-mode emission control. As a consequence, the improvement of motor reliability can be also obtained. By comparing the results, which take into account the inverter and cable arrangements, we can conclude that it is mandatory to consider a more complete topology. In this process, besides the inverter configuration, the installation geometry assumes an important role to achieve the electromagnetic compatibility (EMC).

The proposed solution can be a complementary one and helpful to reduce the size and weight of passive filters. The common-mode current is minimized through the return of the non desired signals back to their source, keeping them in the main circuit. Future works shall evaluate the contribution of the configuration in the amount of differential-mode currents. The next steps of this research shall take care of EMI tests on a prototype, in order to validate the simulations.

Besides the benefits of this topology, an analysis of viability shall also be made, in order to evaluate the potentiality of this solution. It was shown that the motor can be obtained from a conventional three-phase motor with multiple voltages connections, which means no extra costs for this device development. In the other hand, because the inverter has the double of semiconductors, it 
shall cost more compared against a conventional threephase one, with similar power rating. However, it must be observed that each semiconductor will conduct half of the current, allowing the use of smaller IGBT's; surely, costs won't double. When compared with an active commonmode cancellation topology, the cost difference tends to be even smaller. Also, low power inverters, in which control has a significant participation in cost, tend to have a smaller price increase. The same shall happen in high power ones. In those, usually the parallelism of IGBT's is applied, and so, won't have a significant cost increase. No wonder that the solution may be implemented at same cost as a conventional inverter. So, costs shall not be a big issue and the solution shall be affordable for new installations. Existing installations shall be a major barrier due to the need of substitution of the entire system.

The applicability of this solution shall find in transportation segment a good potential for development. Traction systems: railways and, more than this, electric and hybrid-electric vehicles are not a barrier and shall benefit themselves from the EMC related issues. Differently from industry, where many of the inverters and motors are ordered to replace existing ones, in electrical vehicles they are acquired to supply new drive systems. Also, size and weight reductions are important criteria for equipment design and, surely, their reduction in filters will be superior to the increase in inverter power module, in the proposed topology. Besides this, the perspective for the future is a huge growth of the number of electric and hybrid-electric vehicles. Following this growth, EMC concerning issues shall increase, once that those applications shall contribute for the EMI much more than the static industrial installations. In case of vehicles, the area of interference will not be fixed and delimited but will be in any place where a vehicle can reach.

\section{Acknowledgement}

This work was partially supported by CNPq (PQ 307867/2011-0). Likewise, the authors also acknowledge ABB Ltda, Brazil and the Centro Tecnológico da Marinha in São Paulo (CTMSP).

\section{References}

[1] Skibinski, G.L.; Kerkman, R.J.; Schlegel, D.; , "EMI emissions of modern PWM AC drives," Industry Applications Magazine, IEEE, vol.5, no.6, pp.47-80, Nov/Dec 1999

[2] Ogasawara, S.; Ayano, H.; Akagi, H.; "Measurement and reduction of EMI radiated by a PWM inverter-fed AC motor drive system," Industry Applications, IEEE Transactions on, vol.33, no.4, pp.1019-1026, Jul/Aug 1997

[3] Mutoh, N.; Kanesaki, M.; Nakashima, J.; Ogata, M.; "A new method to control common-mode currents focusing on commonmode current paths produced in motor drive systems," Industry Applications Conference, 2003. 38th IAS Annual Meeting. Conference Record of the, vol.1, no., pp. 459- 466 vol.1, 12-16 Oct. 2003

[4] Akagi, H.; Doumoto, T.; , "A passive EMI filter for preventing high-frequency leakage current from flowing through the grounded inverter heat sink of an adjustable-speed motor drive system," Industry Applications, IEEE Transactions on , vol.41, no.5, pp. 1215- 1223, Sept.-Oct. 2005

[5] Julian, A.L.; Oriti, G.; Lipo, T.A.; , "Elimination of commonmode voltage in three-phase sinusoidal power converters," Power Electronics, IEEE Transactions on, vol.14, no.5, pp.982989, Sep 1999

[6] Consoli, A.; Oriti, G.; Testa, A.; Julian, A.L.; "Induction motor modeling for common-mode and differential mode emission evaluation," Industry Applications Conference, 1996. Thirty-First IAS Annual Meeting, IAS '96., Conference Record of the 1996 IEEE, vol.1, no., pp.595-599 vol.1, 6-10 Oct 1996

[7] Cacciato, M.; Consoli, A.; Scarcella, G.; Testa, A.; , "Reduction of common-mode currents in PWM inverter motor drives," Industry Applications, IEEE Transactions on , vol.35, no.2, pp.469-476, Mar/Apr 1999

[8] Di Piazza, M.C.; Tine, G.; Vitale, G.; "An Improved Active Common-Mode Voltage Compensation Device for Induction Motor Drives," Industrial Electronics, IEEE Transactions on, vol.55, no.4, pp.1823-1834, April 2008

[9] Paul, Clayton R.; Introduction to electromagnetic compatibility; 2nd ed, John Wiley \& Sons, Inc, 2006

[10] Hanigovszki, N.; Landkildehus, J.; Spiazzi, G.; Blaabjerg, F.; , "An EMC evaluation of the use of unshielded motor cables in AC adjustable speed drive applications," Power Electronics, IEEE Transactions n , vol.21, no.1, pp. 273- 281, Jan. 2006 